\title{
Magnetic resonance ultrashort echo time spin-echo imaging of the deepest layers of articular cartilage
}

\author{
GAO Song ${ }^{1 *}$, DU Jiang ${ }^{2}$, WANG Fei ${ }^{1} \&$ BAO ShangLian ${ }^{3}$ \\ ${ }^{1}$ Medical Physics Department, Peking University Health Science Center, Beijing 100191, China; \\ ${ }^{2}$ Department of Radiology, University of California, San Diego, CA 92103-8756, USA; \\ ${ }^{3}$ Medical Physics and Engineer Laboratory, School of Physics, Peking University, Beijing 100875, China
}

Received April 6, 2013; accepted June 3, 2013; published online June 7, 2013

Citation: Gao S, Du J, Wang F, et al. Magnetic resonance ultrashort echo time spin-echo imaging of the deepest layers of articular cartilage. Sci China Life Sci, 2013, 56: 672-674, doi: 10.1007/s11427-013-4510-4

Magnetic resonance imaging (MRI) has emerged as an invasive radiologic technique to assess and characterize cartilage lesions in the setting of injury and degenerative joint disease. However, most of the currently available clinical and research MRI techniques, including proton-density weighted fast spin echo (FSE) [1], T2-weighted FSE [2], T2 mapping [3], and steady state free precession imaging [4] have focused on the superficial layers of cartilage. There is a growing interest in the deepest layers of articular cartilage, including the calcified and deep radial layers located just superficial to the subchondral bone $[5,6]$. There has been an emphasis on the role of lesions in the deep radial and calcified layers of cartilage in the pathogenesis of osteoarthritis $[6,7]$. The lack of imaging evaluation of the deep layers of cartilage stems largely from the fact that it is technically difficult to image.

Conventional MRI pulse sequences with echo times (TE) of $1 \mathrm{~ms}$ or greater provide little or no detectable signal from many tissues and tissue components that have very short T2 relaxation times, such as calcified cartilage, menisci, tendons, ligaments and some forms of soft tissue calcification [8]. By using half-sinc radiofrequency (RF) pulses, radial mapping of $k$-space, rapid transmit/receive switching, and variable rate selective excitation, nominal TEs as short as $8 \mu$ s have been achieved with ultrashort TE (UTE) imaging $[9,10]$. Therefore, the UTE pulse sequences make it possible to directly

*Corresponding author (email: gaoss@ hsc.pku.edu.cn) image tissues with very short $\mathrm{T} 2$ and their adjacent tissues [11]. Although short T2 tissues are detectable with UTE sequences, positive visualization of deep layers of cartilage is limited by the presence of high signals from long T2 water and fat.

The dual-echo gradient-echo UTE acquisition has been used previously to suppress long T2 signals in knee cartilage two-dimensional (2D) imaging [8]. With this approach, the second echo is subtracted from the first one, which is equivalent to band pass filtering. This selectively suppresses the signal from the longer T2 components, and typically provides high contrast in the short $\mathrm{T} 2$ range. However, the 2D UTE free induction decay (FID) acquisition is sensitive to eddy currents, gradient anisotropy and timing errors [12]. The latter echoes in this acquisition are sensitive to off-resonance effects. Therefore, we developed a dual-echo spin echo UTE acquisition approach. This letter describes the development and implementation of dual-echo spin-echo UTE sequences, and testing its relative efficacy in terms of signal-to-noise ratio (SNR) and contrast-to-noise ratio (CNR) in imaging deep radial and calcified layers of articular cartilage.

\section{Materials and methods}

As shown in Figure 1, the multi-echo spin-echo UTE acquisition technique was developed where a spin-echo image 


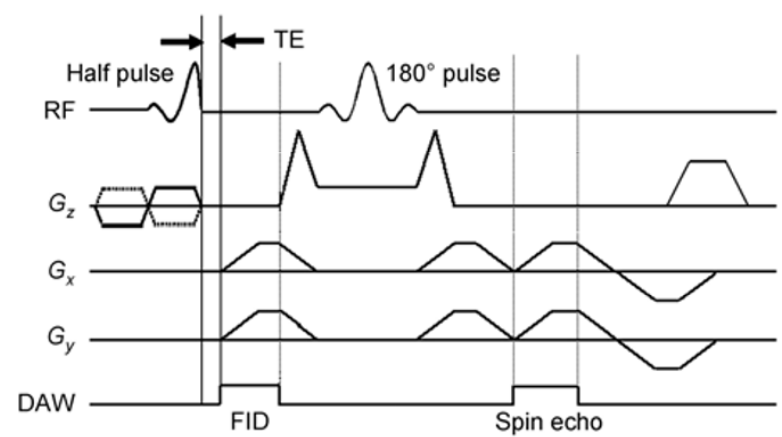

Figure 1 Pulse sequence diagram for 2D dual-echo spin-echo UTE imaging.

was generated by introducing a refocusing $180^{\circ} \mathrm{RF}$ pulse following UTE FID acquisition. Spin-echo data acquisition starts after the refocusing gradient so that the same $k$-space trajectory can be used for both FID and spin-echo on-line image reconstruction.

The performance of the proposed 2D dual-echo spinecho UTE sequence was illustrated by experiments on a $3 \mathrm{~T}$ GE Signa TwinSpeed scanner (GE Healthcare Technologies, Milwaukee, Wisconsin, USA). A quadrature knee coil was used for volunteer knee cartilage imaging. The imaging plane was chosen to be perpendicular to the deep layers of cartilage to minimize partial volume effects. A field of view (FOV) of 8-16 cm was employed with a slice thickness of $1.7-2.5 \mathrm{~mm}$. Multiple interleaved slices were acquired using a TR of 200-600 ms, a flip angle of $60^{\circ}, 511$ half projections, a $62.5 \mathrm{kHz}$ bandwidth, and a 50\%-200\% slice gap. During reconstruction, the $k$-space data were first regridded onto a $512 \times 512$ Cartesian grid using a Kaiser-Bessel kernel, followed by an inverse two-dimensional fast Fourier transformation to generate UTE images. For quantitative assessment of the quality of spin-echo and gradient-echo UTE images, both SNR and CNR measurements were performed. CNR between the deep layers of cartilage and superficial layers of cartilage and fat were calculated as their signal difference over background noise.

\section{Results}

Preliminary results show that UTE spin-echo acquisition provides significantly better image contrast between deep and superficial layers of cartilage $(\mathrm{CNR}=8.7)$ than UTE gradient echo acquisition $(\mathrm{CNR}=4.9)$. Conventional UTE FID acquisition provides the highest mean SNR of 56.5 for deep layers of cartilage. Echo subtraction significantly increased the image contrast between the deep layers and superficial layers of cartilage from 6.1 to 11.8 . However, contrast between deep layers of cartilage and fat is still low.

The $180^{\circ} \mathrm{RF}$ pulse in a spin-echo UTE sequence refocuses the long T2 water and fat spins, and is therefore less sensitive to off-resonance effects. The subtraction image from UTE spin-echo acquisition significantly improved CNR over that from dual-echo gradient-echo acquisition. However, this technique requires a larger slice gap because a broader $180^{\circ} \mathrm{RF}$ pulse is required to more accurately refocus the broad slice profile that resulted from a half pulse excitation. A large flip angle close to $90^{\circ}$ and a longer TR is also helpful.

\section{Discussion}

Subtraction of the spin-echo image from the first FID image may result in better suppression of long T2 water and fat signals, thus improving the delineation of the deep layers of cartilage. Compared with the spin-echo UTE sequence, gradient-echo UTE sequence is sensitive to eddy currents, field homogeneity and gradient non-linearity. Half pulse excitation requires the summation of two acquisitions with the slice selection gradient polarity reversed, so that a conventional slice profile is formed. Gradient profile distortion may result in improper weighting of the excitation and mismatch between the two acquisitions with non-ideal cancellation of the imaginary parts of the complex signals and out-of-slice signal contamination. Corrections of the residual slice-select gradients and time-varying main field $B_{0}(t)$ caused by eddy currents are helpful in reducing out-of-slice signal contamination.

The disadvantage of multiple-echo spin-echo UTE sequences used in this study is that it is a $2 \mathrm{D}$ acquisition with a slice thickness of $1.7-2.5 \mathrm{~mm}$ and a gap of $50 \%-200 \%$ slice thickness. Partial volume effects may significantly degrade the depiction of lesions, which may improve using three-dimensional UTE acquisition [13].

In conclusion, the 2D UTE sequence combined with multiple-echo spin-echo acquisition, with later-echo subtraction, can depict either the superficial layers or the deep layers of knee cartilage on a conventional clinical scanner with a degree of delineation previously not possible. UTE imaging with these modifications show substantial potential of being a powerful radiologic modality for the imaging of cartilage injury and osteoarthritic diseases.

This work was partially supported by the National Natural Science Foundation of China (81171330).

1 Sonin A H, Pensy R A, Mulligan M E, et al. Grading articular cartilage of the knee using fast spin-echo proton density-weighted MR imaging without fat suppression. Am J Roentgenol, 2002, 179: 1159-1166

2 Bredella M A, Tirman P F, Peterfy C G, et al. Accuracy of T2-weighted fast spin-echo MR imaging with fat saturation in detecting cartilage defects in the knee: comparison with arthroscopy in 130 patients. AJR Am J Roentgenol, 1999, 172: 1073-1080

3 Mosher T J, Dardzinski B J. Cartilage MRI T2 relaxation time mapping: overview and applications. Sem Musculoskel Radiol, 2004, 8: $355-368$ 
4 Kijowski R, Lu A, Block W, et al. Evaluation of the articular cartilage of the knee joint with vastly undersampled isotropic projection reconstruction steady-state free precession imaging. J Magn Res Imag, 2006, 24: 168-175

5 Martel-Pelletier J, Lajeunesse D, Fahmi H, et al. New thoughts on the pathophysiology of osteoarthritis: one more step toward new therapeutic targets. Curr Rheumatol Rep, 2006, 8: 30-36

6 Ferguson V L, Bushby A J, Boyde A. Nanomechanical properties and mineral concentration in articular calcified cartilage and subchondral bone. J Anatomy, 2003, 203: 191-202

7 Muir P, McCarthy J, Radtke C, et al. Role of endochondral ossification of articular cartilage and functional adaptation of the subchondral plate in the development of fatigue microcracking of joints. Bone, 2006, 38: 342-349

8 Robson M D, Gatehouse P D, Bydder M, et al. Magnetic resonance: an introduction to ultrashort TE (UTE) imaging. J Comp Ass Tomogr,
2003, 27: 825-846

9 Du J, Corbeil J, Znamirowski R, et al. Direct imaging and quantification of carotid plaque calcification. Magn Reson Med, 2011, 65: 1013-1020

10 Bao S L, Du J, Gao S. Review of the ultrashort echo time magnetic resonance imaging of cortical bone. Acta Phys Sin, 2013, 62: 088701

11 Bae W C, Chen P C, Chung C B, et al. Quantitative ultrashort echo time (UTE) MRI of human cortical bone: correlation with porosity and biomechanical properties. J Bone Min Res, 2012, 27: 848-857

12 Wansapura J P, Daniel B L, Pauly J, et al. Temperature mapping of frozen tissue using eddy current compensated half excitation RF pulses. Magn Reson Med, 2001, 46: 985-992

13 Rahmer J, Bornert $\mathrm{P}$, Groen $\mathrm{J}$, et al. Three-dimensional radial ultrashort echo-time imaging with T2 adapted sampling. Magn Reson Med, 2006, 55: 1075-1082

Open Access This article is distributed under the terms of the Creative Commons Attribution License which permits any use, distribution, and reproduction in any medium, provided the original author(s) and source are credited. 